\title{
A Novel Particle Swarm Optimization with Passive Congregation via Chaotic Sequences
}

\author{
Reza Gholipour, Hamed Mojallali, and Seyed Mohammad Kazem Akhlaghi
}

\begin{abstract}
This paper proposes a novel particle swarm optimization with passive congregation (PSOPC) algorithm based on the chaos phenomenon. The proposed Chaotic PSOPC (CPSOPC) algorithm utilizes chaotic sequences to improve the global searching by escaping the local optima. This algorithm overcomes the disadvantages of premature convergence in the PSOPC. We compared the proposed algorithm with PSOPC for eight benchmark functions with 10 and 20 dimensions. Simulation results indicate that the CPSOPC improves the global convergence of PSOPC. Furthermore, the proposed algorithm improves the accuracy and efficiency of searches compared with PSOPC and increases the convergence speed of PSOPC.
\end{abstract}

Index Terms-Chaotic sequences, particle swarm optimization, passive congregation, logistic map.

\section{INTRODUCTION}

Swarm Intelligence (SI) methods are based around the study of collective behavior in decentralized, self-organized systems. SI systems are typically made up of a population of simple agents interacting locally with one another and with their environment. Although there is no centralized control structure dictating how individual agents should behave, local interactions between such agents often lead to the emergence of a global behavior.

Two of the most successful SI techniques modeled on the behavior of natural systems are ant colony optimization (ACO) proposed by Dorigo and Gambardella [1] and particle swarm optimization (PSO) proposed by Kennedy and Eberhart [2].

In ACO artificial ants build solutions by traversing a problem space. Similar to real ants, they deposit artificial pheromone on the workspace in a manner that makes it possible for future ants to build better solutions. In real ant colonies the pheromone is used to find the shortest path to food. Using ACO, finite size colonies of artificial ants communicate with each other via artificial pheromones to find quality solutions to optimizations problems. ACO has been applied to a wide range of optimization problems such as the traveling salesman problem, and routing and load balancing in packet switched network [1].

The PSO approach utilizes a population based stochastic

Manuscript received October 12, 2012; revised November 14, 2012.

Reza Gholipour is with the Faculty of Electrical and Computer Engineering, Babol (Noushirvani) University of Technology, Babol, Iran (e-mail: reza_gh_08@yahoo.com).

HamedMojallali is with the Electrical Engineering Department, Faculty of Engineering, University of Guilan, Rasht, Iran (e-mail: mojallali@guilan.ac.ir).

Seyed Mohammad KazemAkhlaghi is with the Faculty of Mechanical Engineering, Babol (Noushirvani) University of Technology, Babol, Iran (e-mail: smk_akhlaghi@yahoo.com). optimization algorithm [2]. It was inspired from the computer simulation of the social behavior of bird flocking by Reynolds [3]. In PSO, a set of randomly generated agents propagate in the design space towards the optimal solution over a number of iterations. Each agent has a memory of its best position and the swarm's best solution. PSO is also easy to implement and the fact that no gradient information is required makes it a good candidate for a wide variety of optimization problems [4]. Recently, PSO has been successfully used in the optimization of many engineering fields. ZaferBingul ,OguzhanKarahan, introduced the PSO based tuning method for Fuzzy Logic Controller(FLC) to control the 2 DOF robot trajectory[5].Acharjee and Goswami developed a robust, nondivergent power flow method by using PSO technique[6]. HassenT.Dorrah et al. proposed PSO-BELBIC for two-coupled distillation column process. The PSO_BELBIC improves the time domain parameters of all loops of the process[7]. PSO and its variants widely are applied for tuning of PID controller parameters [8]-[12].

In standard PSO and its variants like: global version of SPSO (GPSO), local version of SPSO (LPSO) and PSO with a constriction factor (CPSO), exchange of information between the particles is low and particles will capture in the local optimum. In PSOPC, by introducing of passive congregation, the exchange of information between particles arises insignificantly. It leads to an improvement in PSO performance [13].

In standard PSO and PSOPC, all particles have the tendency to follow the current best position that may be a local optimum or a position near the local optimum, so that all particles will concentrate to a small region and cannot search in global solution space again. Thus, particles will capture in the local optimum and the premature convergence phenomena occur.

In order to, improve the PSOPC performance, a Chaotic PSOPC is delivered in this paper. In this method, adding chaotic sequences with ergodic, irregular, dynamic and stochastic properties in PSOPC the global convergence is improved.

The rest of the paper is organized as follows: Section 2 describes typical PSO. In section 3 PSOPC is described. Section 4 presents the proposed CPSOPC algorithm. Section 5 expresses the result from CPSOPC while compared with other algorithms for benchmark functions. Finally, the last section gives an abbreviated conclusion about this study.

\section{Particle SWARm Optimization}

The PSO is an evolutionary computational model, a stochastic search technique based on swarm intelligence. Social behavioral pattern of organisms such as bird flocking 
and fish schooling inspired them to look into the effect of collaboration of species when obtaining their goals as a group. Dynamics of bird flocking resulted in the possibilities of utilizing this behavior as an optimization tool. These have been used to solve a range of optimization problems.

Suppose that a swarm contains $m$ particles moving around in a $d$-dimensional search space. The ith particle at the $t$ th iteration has a position $X_{i}(t)=\left(X_{i 1}(t), X_{i 2}(t), \ldots, X_{i d}(t)\right)$, which is a feasible solution of an optimal problem, a velocity $V_{i}(t)=\left(V_{i 1}(t), V_{i 2}(t), \ldots, V_{i d}(t)\right)$, and the best solution ever achieved so far by itself, individual best position $P_{\text {best }}$, which is represented by $P_{i}(t)=\left(P_{i 1}(t), P_{i 2}(t), \ldots, P_{i d}(t)\right)$. The best solution ever achieved so far by the whole swarm, global best position $G_{\text {best }}$, which is represented by $P_{g}(t)=\left(P_{g 1}(t), P_{g 2}(t), \ldots, P_{g d}(t)\right)$. Then, the velocity andthe position of the ith particle in the $k$ th dimension at the next iteration will be calculated as:

$$
\begin{gathered}
V_{i k}(t+1)=w * V_{i k}(t)+c_{1} * r_{1} *\left(P_{i k}(t)-X_{i k}(t)\right) \\
+c_{2} * r_{2} *\left(P_{g k}(t)-X_{i k}(t)\right) \\
X_{i k}(t+1)=X_{i k}(t)+V_{i k}(t+1)
\end{gathered}
$$

where $w$ is inertia weight, $c_{1}$ and $c_{2}$ are two positive constants, called cognitive learning rate and social learning rate, respectively, and $r_{1}$ and $r_{2}$ are two uniformly random numbers from interval $[0,1]$.

A linearly decreasing inertia weight [14], [15] was first introduced by Shi and Eberhart in 1998. At the beginning of the searching process, a larger inertia factor is applied for global exploration. During the search, inertia weight is becoming smaller and smaller for local exploitation, which leads to significant improvement in the performance of classical PSO. The linearly decreasing inertia weight is modified as:

$$
w(t)=w_{\max }-\frac{w_{\text {max }}-w_{\text {min }}}{t_{\max }} * t
$$

And the velocity is modified as:

$$
\begin{aligned}
& V_{i k}(t+1)=\mathrm{w}(t) * V_{i k}(t)+c_{1} * r_{1} *\left(P_{i k}(t)-X_{i k}(t)\right) \\
& +c_{2} * r_{2} *\left(P_{g k}(t)-X_{i k}(t)\right)
\end{aligned}
$$

where $w_{\text {max }}$ is the initial inertia weight, $w_{\text {min }}$ is the final inertia weight, and $t_{\max }$ is the number of maximum iteration. Normally, $w_{\max }$ is set to 0.9 and $w_{\min }$ is set to 0.4 .

\section{Particle SWARm Optimization With Passive CONGREGATION}

The PSO can be enhanced by one type of social behaviors such as bird flocking, fish schooling and insects swarming, which are considered as congregation. This behavior is connected with grouping by social forces which is the source of attraction .The congregation includes active congregation and passive congregation .The latter is an attraction of an individual to other group members but not a display of social behavior [13]. Fish schooling is one of the representative types of passive congregation and the PSO is inspired by it .Adding the passive congregation model to the SPSO may increase its performance .He et al. proposed a hybrid PSO with passive congregation (PSOPC) as fallows [13]:

$$
\begin{gathered}
V_{i Q}(t+1)=w V_{i Q}(t)+c_{1} r_{1}\left(P_{i Q}(t)-X_{i Q}(t)\right)+ \\
c_{2} r_{2}\left(P_{g Q}(t)-X_{i Q}(t)\right)+c_{3} r_{3}\left(R_{i Q}(t)-X_{i Q}(t)\right) \\
X_{i Q}(t+1)=X_{i Q}(t)+V_{i Q}(t+1)
\end{gathered}
$$

where $R_{i}$ is a particle selected randomly from the swarm, $c_{3}$ is the passive congregation coefficient and $r_{3}$ is a uniform random sequences in the range $(0,1): r_{3} \sim U[0,1]$.

\section{ChaOtic Particle SWARM Optimization WITH PASSIVE CONGREGATION}

In the field of engineering, it is well recognized that chaos theory can be applied as a very useful technique in practical application. The chaotic system can be described by a phenomenon, in which a small change in the initial condition will lead to nonlinear change in future behavior, besides that the system exhibits distinct behaviors under different phases, i.e. stable fixed points, periodic oscillations, bifurcations, and ergodicity. Chaos is also a common nonlinear phenomenon with much complexity and is similar to randomness. Chaos is typically highly sensitive to the initial values and thus provides great diversity based on the ergodic property of the chaos phase, which transits every state without repetition in certain ranges. It is generated through a deterministic iteration formula. Due to these characteristics, chaos theory can be applied in optimization.

The Chaotic particle swarm optimization with passive congregation (CPSOPC) algorithm is based on PSOPC and chaotic sequences. In the PSOPC convergence, the parameters $w, c_{1}, c_{2}, r_{1}, r_{2}$ and $r_{3}$ are mainly the effective key factor [13, 16 and 17]. In CPSOPC, sequences generated by the logistic map [18] substitute the random parameters $r_{1}$ and $r_{2}$ in PSOPC. The parameters $r_{1}$ and $r_{2}$ are modified by the logistic map based on the following equation.

$$
\operatorname{Cr}(t+1)=4 \operatorname{Cr}(t)(1-\operatorname{Cr}(t)), \operatorname{Cr}(t) \in[0,1]
$$

In Eq.7, $\operatorname{Cr}(0)$ is generated randomly for each independent run, with $\operatorname{Cr}(0)$ not being equal to $\{0,0.25,0.5,0.75,1\}$. Chaotic sequences $\mathrm{Cr}$ are sensitive to the initial conditions of $\operatorname{Cr}(0)$. The chaotic values of $\mathrm{Cr}$ for $\operatorname{Cr}(0)=0.0001$ and $\operatorname{Cr}(0)=0.00011$ are shown in Fig. 1 .

Dependence of chaotic sequences $\mathrm{Cr}$ to initial conditions is depicted in Figure 1. As it shows, with little change in initial condition of $\mathrm{Cr}(0)$, chaotic sequences $\mathrm{Cr}$ will lead to huge changes. 
The velocity and position update equations for CPSOPC, can be formulated as:

$$
\begin{gathered}
V_{i Q}(t+1)=w V_{i Q}(t)+c_{1} C r_{1}\left(P_{i Q}(t)-X_{i Q}(t)\right) \\
+c_{2} C r_{2}\left(P_{g Q}(t)-X_{i Q}(t)\right)+c_{3} r_{3}\left(R_{i Q}(t)-X_{i Q}(t)\right) \\
X_{i Q}(t+1)=X_{i Q}(t)+V_{i Q}(t+1)
\end{gathered}
$$

where $C r_{1}, C r_{2} \in[0,1]$ are functions based on results of logistic map. The flowchart of proposed algorithm CPSOPC is described as in Fig. 2.

\section{NUMERICAL SIMULATIONS}

\section{A. Benchmark Functions}

In order to test the performance of proposed algorithm on numerical function optimization, eight numerical benchmark functionsare used here.
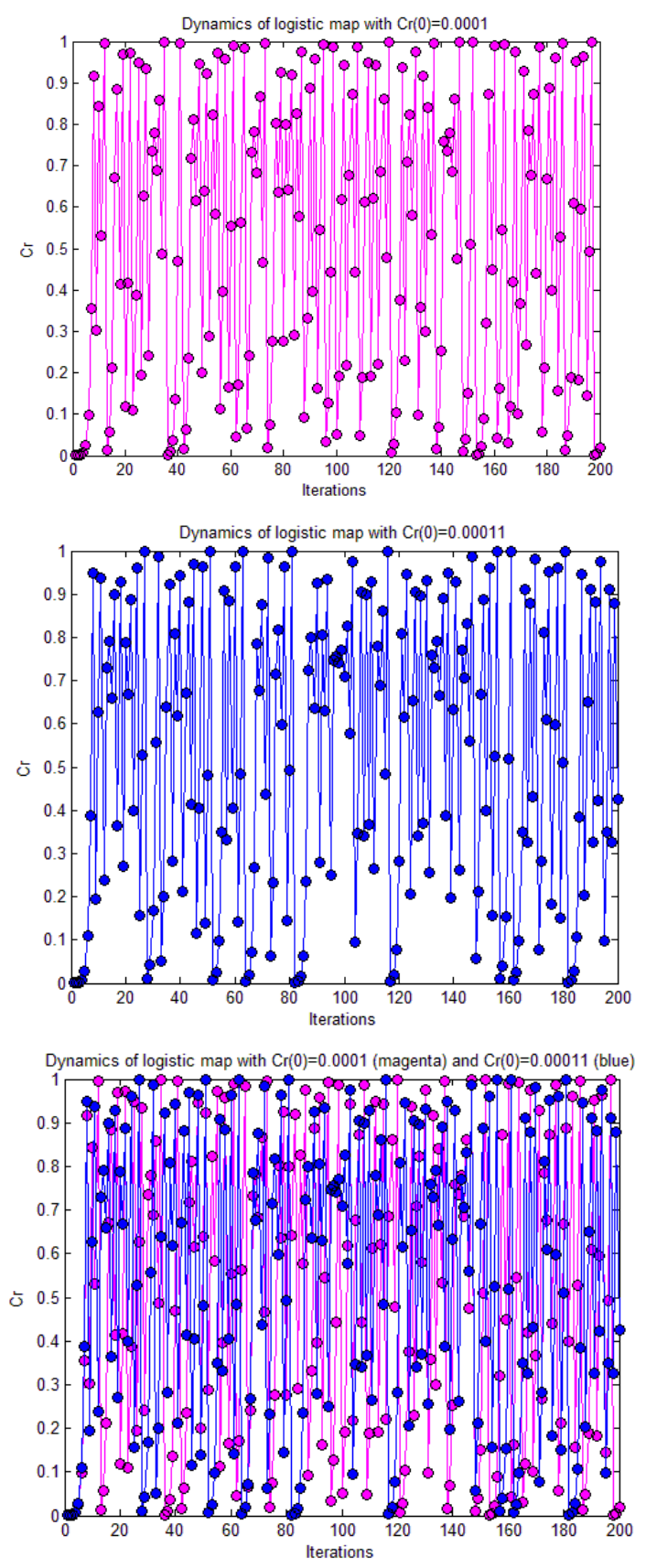

Fig. 1. Dynamics of logistic map
The first functionis the Sphere function described by

$$
f_{1}(\vec{x})=\sum_{i=1}^{D} x_{i}^{2}
$$

where the initial range of $\vec{x}$ is $[-100,100]^{D}$. The minimum solution of the Sphere function is $\vec{x}^{*}=[0,0, \ldots, 0]$ and $f_{1}\left(\vec{x}^{*}\right)=0$.

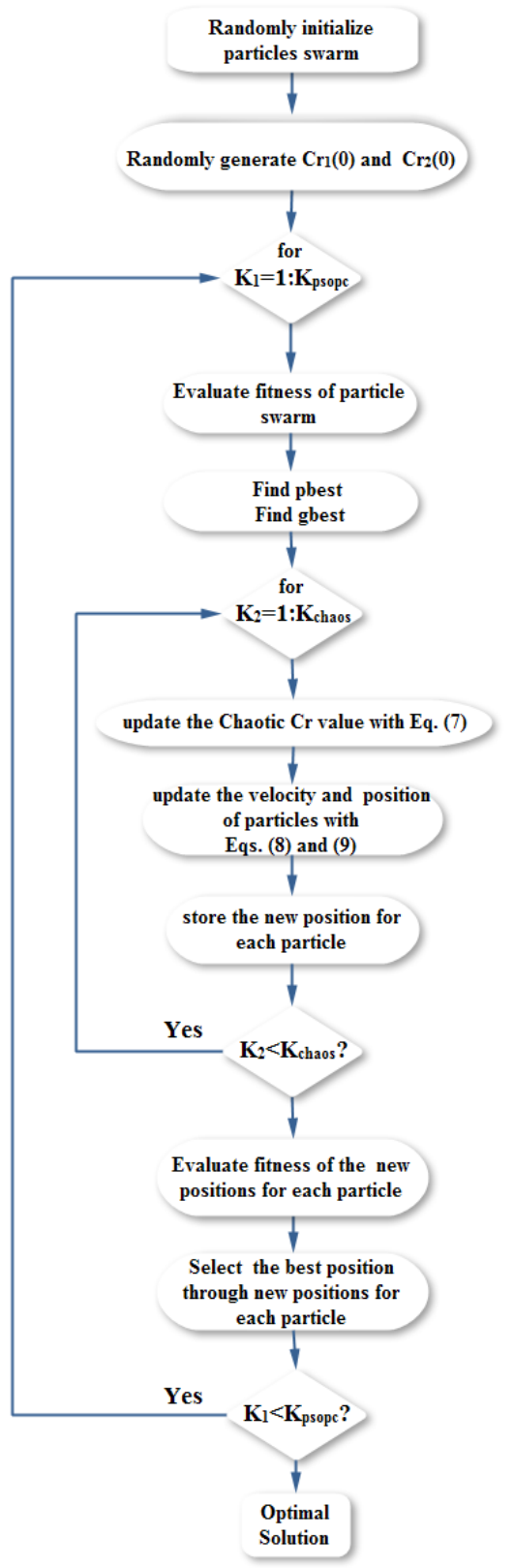

Fig. 2. Flowchart of CPSOPC algorithm

The second function is the Griewank function described by

$$
f_{2}(\vec{x})=\frac{1}{4000}\left(\sum_{i=1}^{D}\left(x_{i}-100\right)^{2}\right)-\left(\prod_{i=1}^{D} \cos \left(\frac{x_{i}-100}{\sqrt{i}}\right)\right)+1
$$

where the initial range of $\vec{X}$ is $[-600,600]^{D}$. The minimum solution of the Griewank function is $\vec{x}^{*}=[100,100, \ldots, 100]$ and $f_{2}\left(\vec{x}^{*}\right)=0$.

The third function isthe Ackley function described by 


$$
\begin{aligned}
& f_{3}(\vec{x})=20+e-20 \exp \left(-0.2 \sqrt{\frac{1}{D} \sum_{i=1}^{D} x_{i}^{2}}\right)- \\
& \exp \left(\frac{1}{D} \sum_{i=1}^{D} \cos \left(2 \pi x_{i}\right)\right)
\end{aligned}
$$

where the initial range of $\vec{x}$ is $[-32.768,32.768]^{D}$.The minimum solution of the Rastrigin function is $\vec{x}^{*}=[0,0, \ldots, 0]$ and $f_{3}\left(\vec{x}^{*}\right)=0$. by

The fourth function is the Rosenbrock function described

$$
f_{4}(\vec{x})=\sum_{i=1}^{D-1} 100\left(x_{i+1}-x_{i}^{2}\right)^{2}+\left(x_{i}-1\right)^{2}
$$

where the initial range of $\vec{x}$ is $[-50,50]^{D}$. The minimum solution of the Rosenbrock function is $\vec{x}^{*}=[1,1, \ldots, 1]$ and $f_{4}\left(\vec{x}^{*}\right)=0$.

The fifth function is the Rastrigin function described by

$$
f_{5}(\vec{x})=\sum_{i=1}^{D}\left(x_{i}^{2}-10 \cos \left(2 \pi x_{i}\right)+10\right)
$$

where the initial range of $\vec{x}$ is $[-5.12,5.12]^{D}$.The minimum solution of the Rastrigin function is $\vec{x}^{*}=[0,0, \ldots, 0]$ and $f_{5}\left(\vec{x}^{*}\right)=0$.

The sixth function is the generalized Schaffer function described by

$$
f_{6}(\vec{x})=0.5+\frac{\sin ^{2}\left(\sqrt{\sum_{i=1}^{D} x_{i}^{2}}\right)-0.5}{\left(1+0.001\left(\sqrt{\sum_{i=1}^{D} x_{i}^{2}}\right)\right)^{2}}
$$

where $\vec{x}=\left[x_{1}, x_{2}, \ldots, x_{D}\right]$, the initial range of $\vec{x}$ is $[-100,100]^{D}$ and $D$ denotes the dimension of the solution space. The minimum solution of the Schaffer function is $\vec{x}^{*}=[0,0, \ldots, 0]$ and $f_{6}\left(\vec{x}^{*}\right)=0$

The seventh function is the Schwefel function described by

$$
\begin{gathered}
f_{7}=418.9829 D-\sum_{i=1}^{D} x_{i} \sin \left(\sqrt{\left|x_{i}\right|}\right) \\
f_{8}=\frac{\pi}{30}\left\{\begin{array}{l}
10 \sin ^{2}\left(\pi y_{1}\right)+\sum_{i=1}^{29}\left(y_{i}-1\right)^{2} \times \\
{\left[1+10 \sin ^{2}\left(\pi y_{i+1}\right)\right]+\left(y_{n}-1\right)^{2}}
\end{array}\right\} \\
+\sum_{i=1}^{30} u\left(x_{i}, 10,100,4\right)
\end{gathered}
$$

where $\vec{x}=\left[x_{1}, x_{2}, \ldots, x_{D}\right]$, the initial range of $\vec{x}$ is $[-500,500]^{D}$ and $D$ denotes the dimension of the solution space. The minimum solution of the Schaffer function is $\vec{x}^{*}=[420.9687,420.9687, \ldots, 420.9687]$ and $f_{7}\left(\vec{x}^{*}\right)=0$
The eighth function is the Penalized function described by where

$$
u\left(x_{i}, a, k, m\right)=\left\{\begin{array}{lc}
k\left(x_{i}-a\right)^{m} & \mathrm{x}_{\mathrm{i}}>\mathrm{a} \\
0 & -\mathrm{a} \leq \mathrm{x}_{\mathrm{i}} \leq \mathrm{a} \\
k\left(-x_{i}-a\right)^{m} & \mathrm{x}_{\mathrm{i}}<-\mathrm{a}
\end{array}\right.
$$

And

$$
y_{i}=1+\frac{1}{4}\left(x_{i}+1\right)
$$

where $\vec{x}=\left[x_{1}, x_{2}, \ldots, x_{D}\right]$, the initial range of $\vec{x}$ is $[-50,50]^{D}$ and $D$ denotes the dimension of the solution space. The minimum solution of the Schaffer function is $\vec{x}^{*}=[-1,-1, \ldots,-1]$ and $f_{8}\left(\vec{x}^{*}\right)=0$.

\section{B. Experimental Setting}

The population size of three algorithms was set at 70 . The acceleration constants $C_{1}$ and $C_{2}$ in CPSOPC and PSO were set 2 and in PSOPC was set 0.5. In both CPSOPC and PSOPC the passive congregation coefficient $C_{3}$ linearly increases from 0.4 to 0.6 . The inertia weight $w$ for three algorithms started from 0.9 and decreased linearly to 0.7 . The number of iteration allowed in each run was set to 500 for dimension 10 and 1000 for dimension 20. All experiments were repeated for 80 runs. In CPSOPC algorithm the number of chaos iteration $K_{\text {chaos }}$ was set to be 15 .

TABLE I: COMPARISON OF ACQUIRED RESULTS FROM CPSOPC WITH PSOPC AND PSO

\begin{tabular}{|l|l|l|l|l|l|l|l|}
\hline \hline \multirow{2}{*}{$\begin{array}{l}\text { Test } \\
\text { function }\end{array}$} & \multirow{2}{*}{ Dim } & CPSOPC & & PSOPC & & PSO & \\
\cline { 2 - 8 } & & Meann & SD & Mean & SD & Mean & SD \\
\hline \multirow{2}{*}{$f_{1}$} & 10 & $5.4397 \mathrm{e}-020$ & $8.6844 \mathrm{e}-020$ & $1.4678 \mathrm{e}-005$ & $4.3686 \mathrm{e}-005$ & 0.0364 & 0.0890 \\
\cline { 2 - 8 } & 20 & $1.0506 \mathrm{-}-011$ & $1.9929 \mathrm{e}-011$ & 168.5687 & 101.7761 & 501.0667 & $2.2361 \mathrm{e}+0003$ \\
\hline \multirow{2}{*}{$f_{2}$} & 10 & 0.0888 & 0.0380 & 0.1350 & 0.0913 & 0.2752 & 0.1804 \\
\cline { 2 - 8 } & 20 & 0.0255 & 0.0172 & 3.5740 & 2.9779 & 4.6275 & 14.0996 \\
\hline \multirow{2}{*}{$f_{3}$} & 10 & $8.4337 \mathrm{e}-010$ & $2.1477 \mathrm{e}-009$ & 0.8005 & 0.9435 & 1.3316 & 0.9721 \\
\cline { 2 - 8 } & 20 & $2.2275 \mathrm{e}-004$ & $2.3721 \mathrm{e}-004$ & 1.4760 & 0.8945 & 5.2445 & 6.5278 \\
\hline \multirow{2}{*}{$f_{4}$} & 10 & 0.0011 & 0.0041 & 45.1296 & 116.2293 & 60.3922 & 118.4356 \\
\cline { 2 - 8 } & 20 & 10.7270 & 1.2380 & 202.7378 & 575.9683 & 246.8969 & 593.4850 \\
\hline \multirow{3}{*}{$f_{5}$} & 10 & 3.4326 & 2.3384 & 8.7629 & 4.8263 & 12.7924 & 8.0170 \\
\cline { 2 - 8 } & 20 & 16.9646 & 12.8535 & 47.7107 & 14.9805 & 51.1067 & 15.9686 \\
\hline \multirow{2}{*}{$f_{6}$} & 10 & 0.0097 & $3.8005 \mathrm{e}-010$ & 0.0269 & 0.0183 & 0.0502 & 0.0219 \\
\cline { 2 - 8 } & 20 & 0.0097 & $1.2488 \mathrm{e}-010$ & 0.1525 & 0.0475 & 0.3632 & 0.0616 \\
\hline \multirow{2}{*}{$f_{7}$} & 10 & 922.9784 & 192.6075 & 970.3547 & 333.5117 & $1.0028 \mathrm{e}+003$ & 276.7715 \\
\cline { 2 - 8 } & 20 & $2.5195 \mathrm{e}+003$ & 438.1564 & $2.7191 \mathrm{e}+003$ & 505.9755 & $2.8186 \mathrm{e}+003$ & $1.0301 \mathrm{e}+003$ \\
\hline \multirow{2}{*}{$f_{8}$} & 10 & $3.0861 \mathrm{e}-020$ & $1.2912 \mathrm{e}-019$ & 0.0456 & 0.1046 & 0.0856 & 0.2419 \\
\cline { 2 - 8 } & 20 & $9.0095 \mathrm{e}-011$ & $2.4246 \mathrm{e}-010$ & 0.6139 & 0.6274 & 1.4461 & 0.8485 \\
\hline \hline
\end{tabular}

\section{Experimental Results}

The experimental results consist of the mean and the standard deviations of the function values found in 80 runs are listed in Table I. From the results of Table1, CPSOPC algorithm outperforms the PSOPC algorithm for all thebenchmark functions. Mean and std illustrate the CPSOPC hasextraordinary convergence and robustness.

Various curves, in Fig. 2-9 illustrate the mean fitness values resulted by the three algorithms with respect to iterations. 

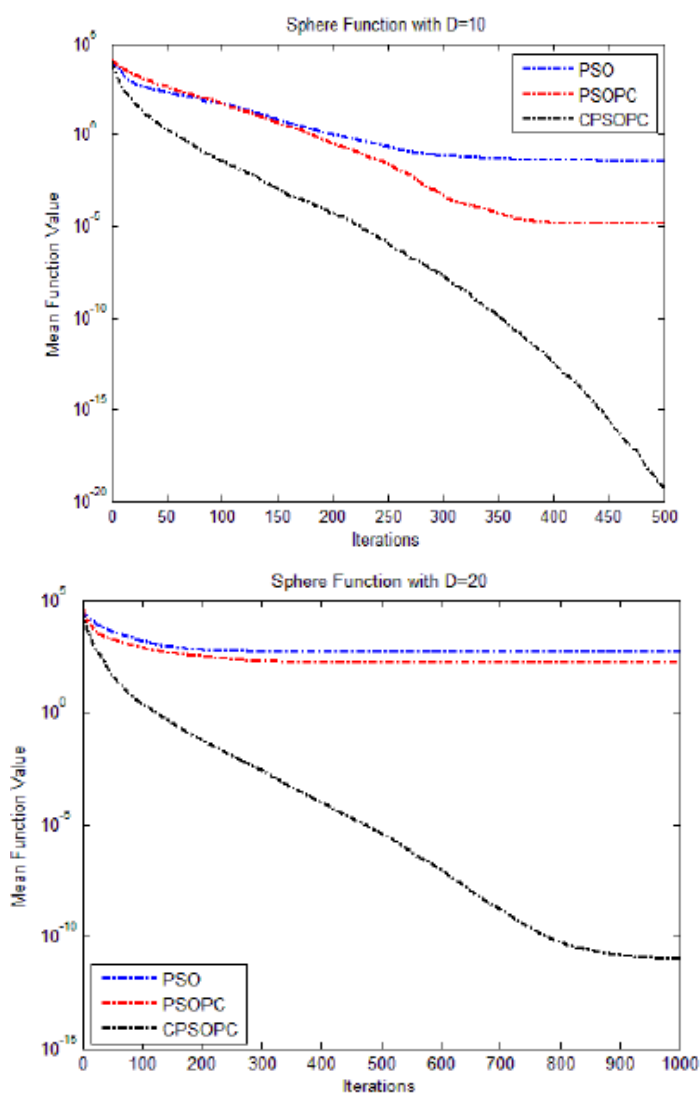

Fig. 2. Convergence curves of CPSOPC, PSOPC and PSO algorithms for the Sphere function for $\mathrm{D}=10$ and $\mathrm{D}=20$
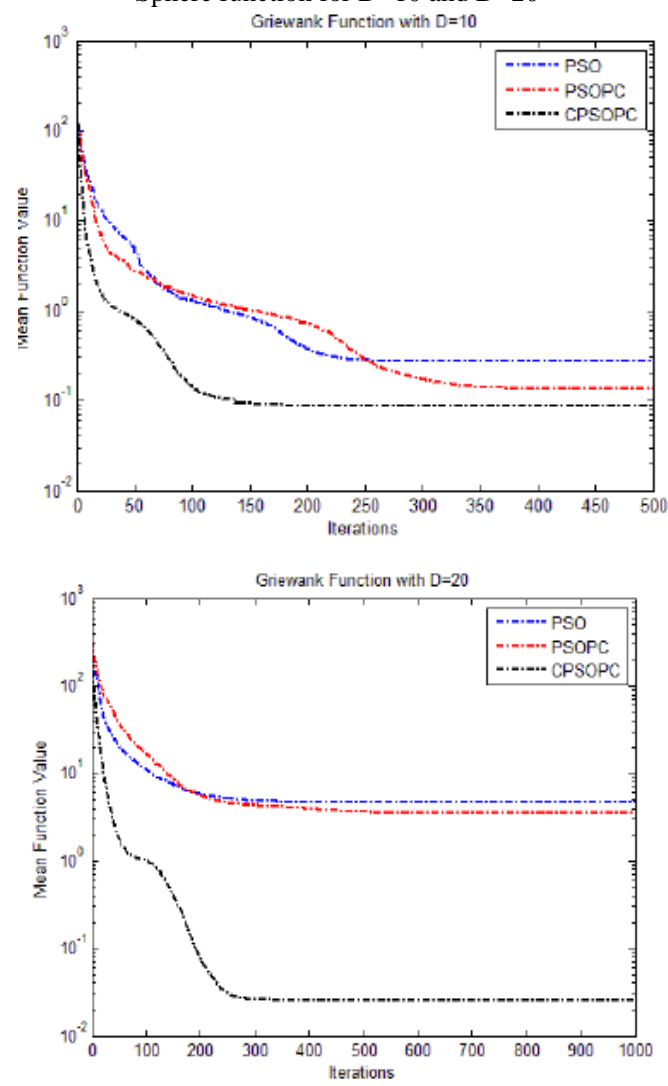

Fig. 3. Convergence curves of CPSOPC, PSOPC and PSO algorithms for the Griewank function for $\mathrm{D}=10$ and $\mathrm{D}=20$

According to Fig. 2-9, the CPSOPC can find the best fitness with fast convergence speed. In fact with adding chaotic sequences to PSOPC, the search behavior is improved and the entrapment of particles in a local optimal is prevented.
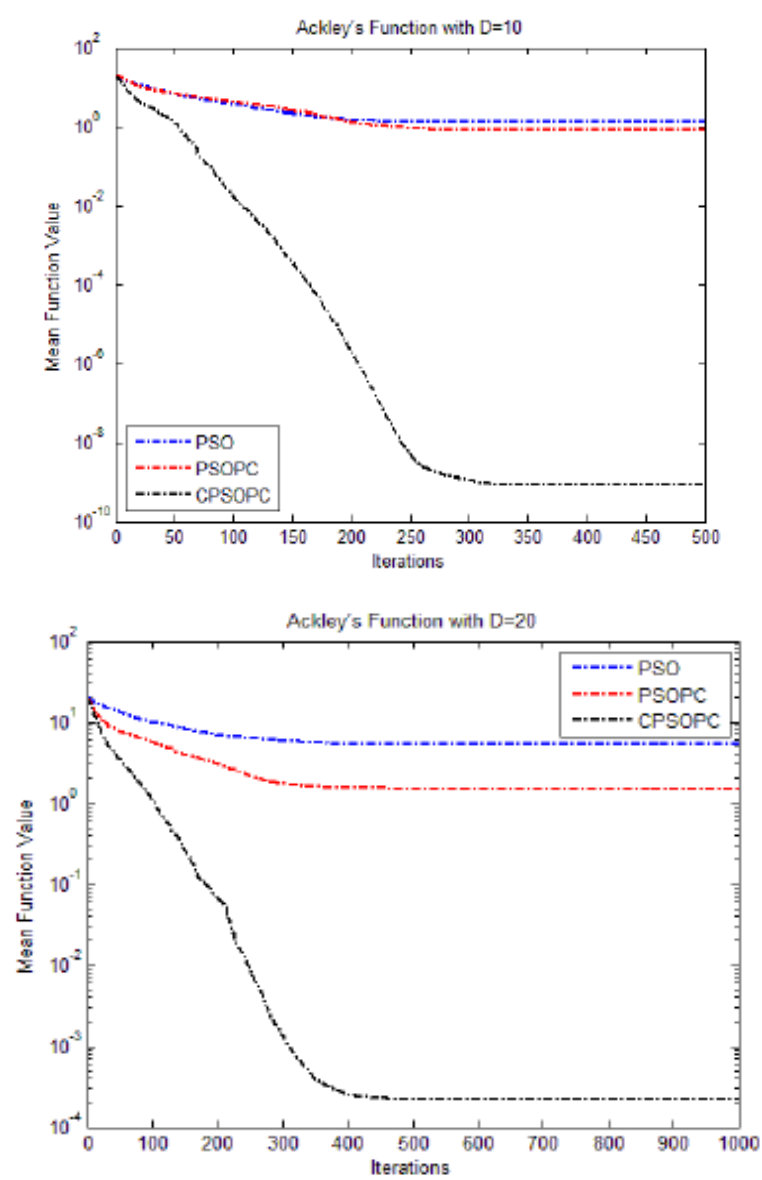

Fig. 4. Convergence curves of CPSOPC, PSOPC and PSO algorithms for the Ackley function for $\mathrm{D}=10$ and $\mathrm{D}=20$
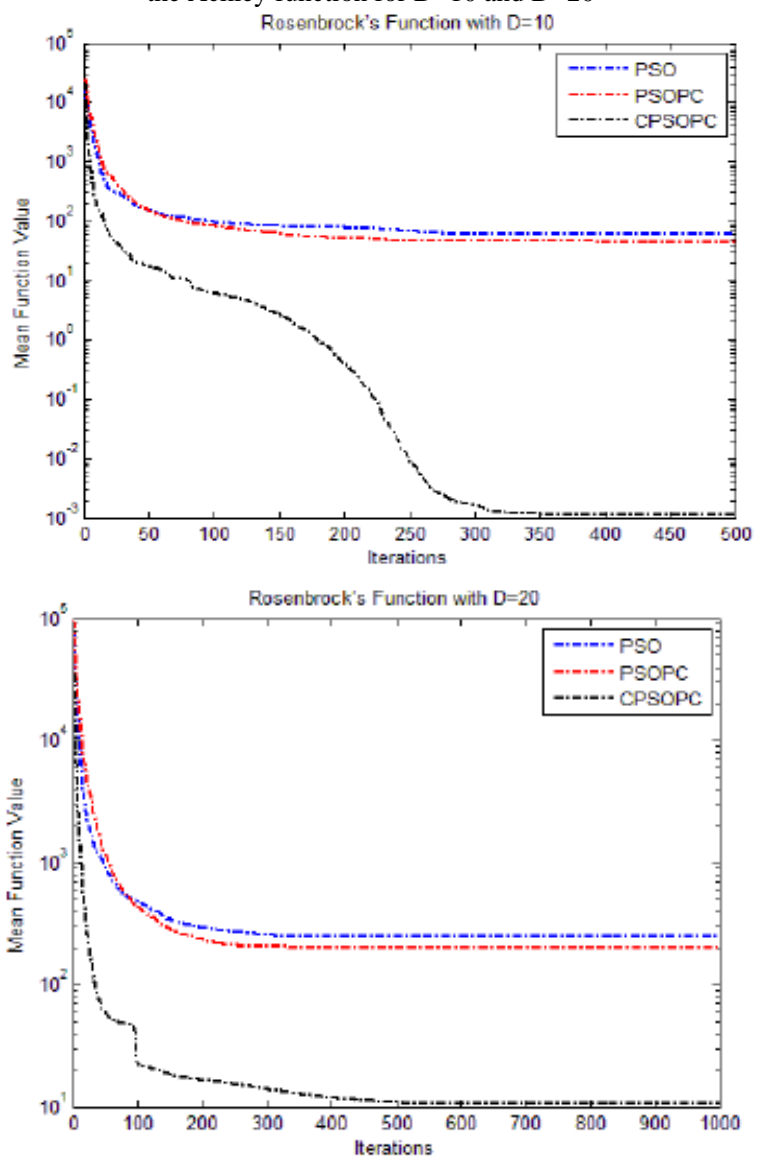

Fig. 5. Convergence curves of CPSOPC, PSOPC and PSO algorithms for the Rosenbrock function for $\mathrm{D}=10$ and $\mathrm{D}=20$ 

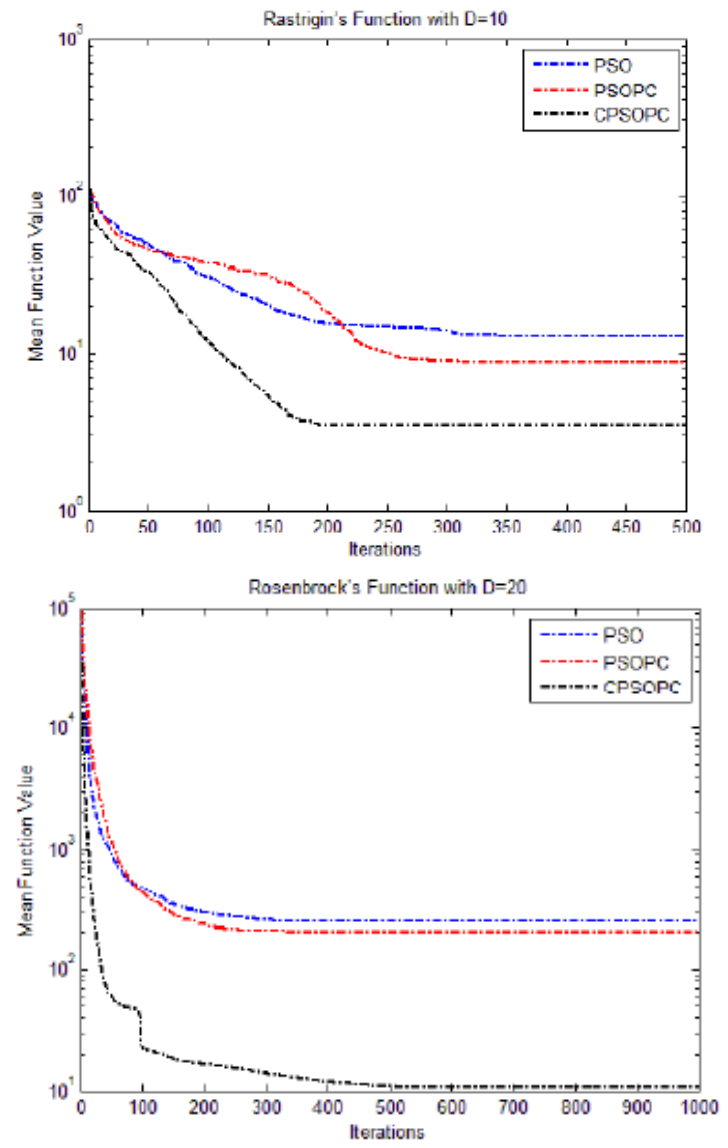

Fig. 6. Convergence curves of CPSOPC, PSOPC and PSO algorithms for the Rastrigin function for $\mathrm{D}=10$ and $\mathrm{D}=20$
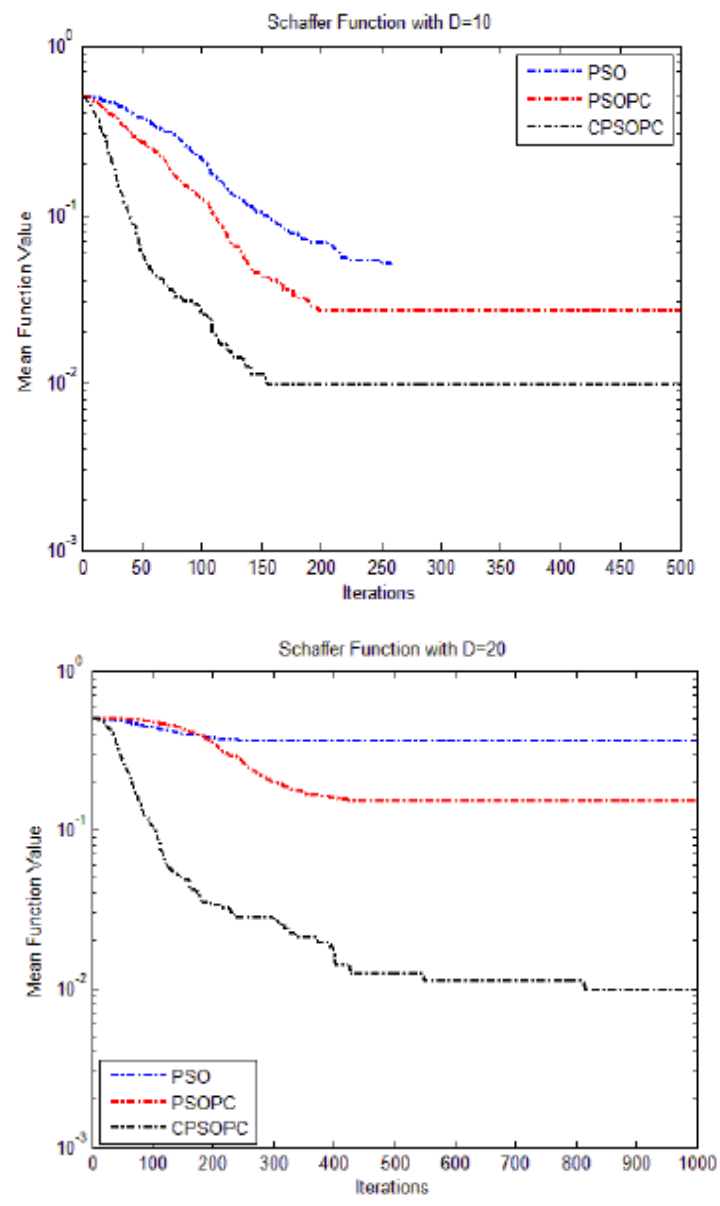

Fig. 7. Convergence curves of CPSOPC, PSOPC and PSO algorithms for the Schaffer function for $\mathrm{D}=10$ and $\mathrm{D}=20$
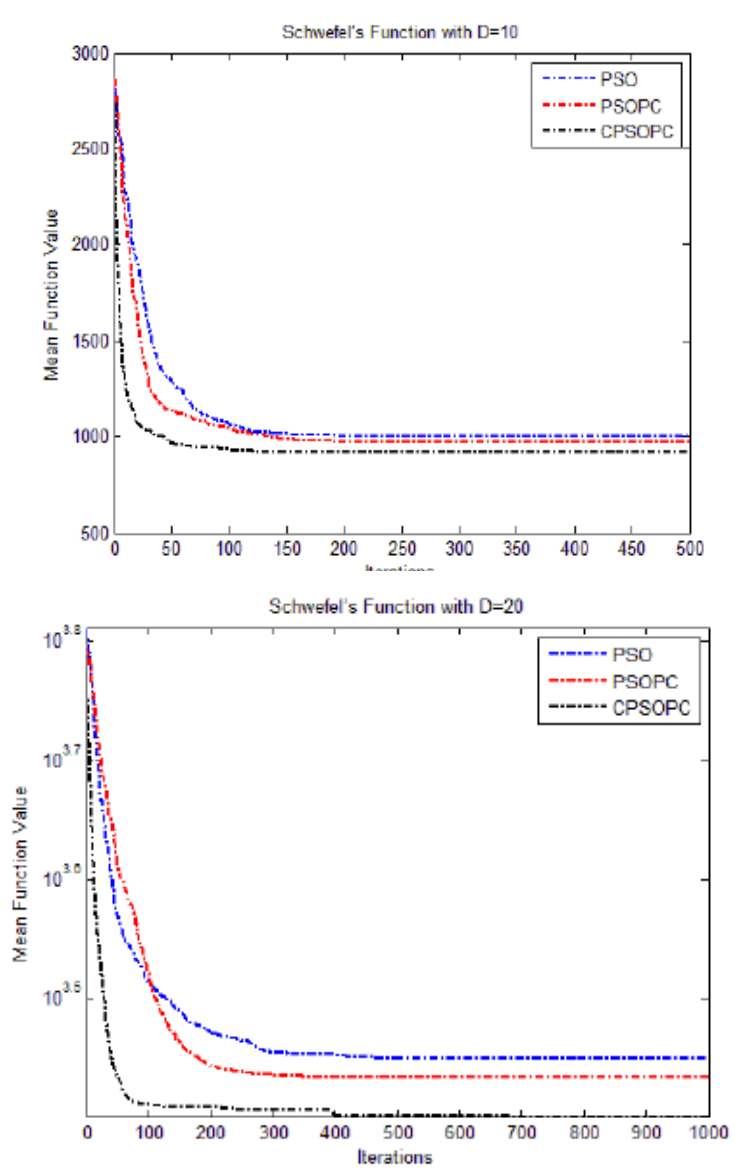

Fig. 8. Convergence curves of CPSOPC, PSOPC and PSO algorithms for the Schwefel function for $\mathrm{D}=10$ and $\mathrm{D}=20$
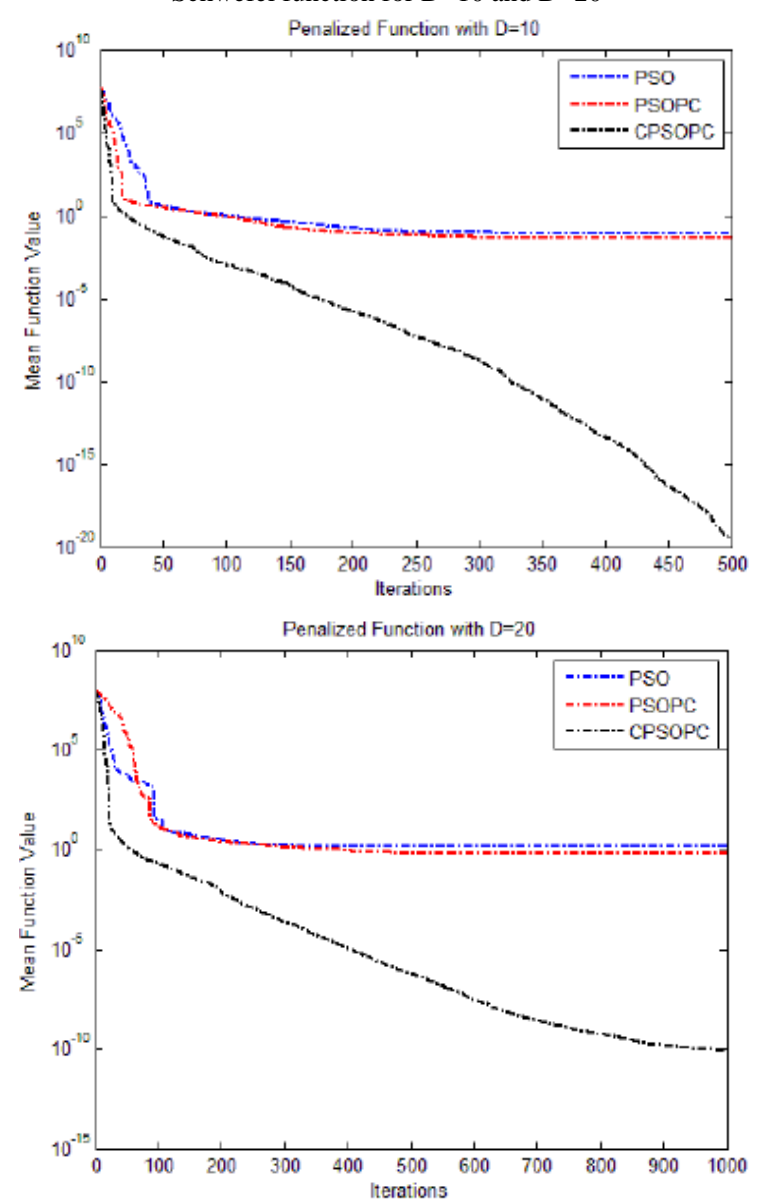

Fig. 9. Convergence curves of CPSOPC, PSOPC and PSO algorithms for the Penalized function for $\mathrm{D}=10$ and $\mathrm{D}=20$ 


\section{Discussion}

In CPSOPC, a chaotic map was embedded to determine the PSOPC parameters $r_{1}$ and $r_{2}$. The PSO and PSOPC parameters $r_{1}$ and $r_{2}$ cannotensure optimal ergodicity in the search space because they are absolutely randomi.e. the $r_{1}$ and $r_{2}$ are generated by alinear congruential generator (LCG) with a random seed. The generated sequence of LCG consists of pseudo-random numbersthat have periodic characteristics [19]. Furthermore, the generated sequence of a logistic map also consists of pseudo-randomnumbers, but there are no fixed points, periodic orbits, or quasi periodic orbits in the behavior of the chaos system [20].

As a result, the system can avoid being entrapment in local optima. In terms of mean fitness values, figures 2-9indicate that CPSOPC outperformed PSOPC on the Sphere, Griewank, Ackley, Rosenbrock, Rastrigin, Schaffer, Schwefel and Penalized functions.

\section{CONCLUSION}

In this paper, a chaotic particle swarm optimization with passive congregation (CPSOPC), based on the particle swarm optimization with passive congregation (PSOPC), and chaotic sequences is presented to improve the premature convergence in the PSOPC method. The Comparison between CPSOPC and PSOPC has shown that the proposed algorithm improves the global search of PSOPC by escaping the local optima. The CPSOPC algorithm tested on eight benchmarkfunctions and results show the efficiency of the proposed algorithm.

\section{REFERENCES}

[1] M. Dorigo and L. M. Gambardella, "Ant Colony System: A Cooperative Learning Approach to the Traveling Salesman Problem,"IEEE Transaction on Evolutionary Computation, pp. 53-66, 1997.

[2] J. Kennedy and R. Eberhart, "Particle swarm optimization,” in Proc. of IEEE Int. Conf. Neural Networks, vol. 4, Perth, Australia,1995, pp. 1942-1948.
[3] C. Reynolds, "Flocks, Herds and Schools: A Distributed Behavioral Model,”Computer Graphics, vol. 21, no.4, pp. 25-34, 1987.

[4] J. Kennedy, R. C. Russell, and Y. Shi, "Swarm Intelligence," The Morgan Kaufmann Series in Evolutionary Computation, 2001.

[5] Z. Bingül and O. Karahan, “A Fuzzy Logic Controller tuned with pso for 2 DOF robot trajectory control," expert systems with Applications, vol. 38, pp.1017-1031, 2011.

[6] P. Acharjee and S. K. Goswami, "A decoupled power flow algorithm using particle swarm optimization technique," Energy Convers Manage, vol. 50, no. 9, pp. 2351-60, 2009.

[7] H. T. Dorrah, A. M. E. Garhy, and M. E. E. Shimy, "PSO_BELBIC Scheme for two_coupled distillation column process," Journal of advanced Research, vol. 2, pp. 73-83, 2011.

[8] H. Fang, L.Chen, and Z. Shen, "Application of an improved pso algorithm to optimal tuning of PID gains for water turbine governor," Energy Conversion and Management, vol. 52, pp. 1763-1770, 2011.

[9] W. D. Chang and S. P. Shih, "PID controller design of nonlinear systems using an improved particle swarm optimization approach," Commun Nonlinear Sci Number Simulat, vol. 15, pp. 3632-3639, 2010.

[10] Y. Wakasa, K. Tanaka, T. Akashi, and Y. Nishimura, "pso_based simultaneous Tuning Method for PID controllers and dead_zone compensators and its application to ultrasonic Motors,” International Journal of Innovative Computing, Information and control (ICIC), vol. 6, no. 10, pp. 4593-4604, 2010.

[11] J. S. Chiou and M. T. Liu, "Numerical Simulation for Fuzzy_PID controllers and helping EP reproduction with pso hybrid algorithm," Simulation Modeling Practice and Theory, vol. 17, pp. 1555-1565, 2009.

[12] T. H. Kim, I. Maruta, and T. Sugie, "Robust PID controller tuning based on the constrained particle swarm optimization," Automatica, vol. 44, pp. 1104-1110, 2008.

[13] S. He, Q. H. Wu, J. Y. Wen, J. R. Saunders, and R. C. Paton, “A particle swarm optimizer with passive congregation,” Bio Systems, vol. 78, pp. 135-147, 2004

[14] Y. Shi and R. C. Eberhart, “A modified particle swarm optimizer,” in Proceedings of the IEEE international conference on evolutionary computation, 1998, pp. 69-73.

[15] Y. Shi and R. C. Eberhart, "Empirical study of particle swarm optimization," in Proceedings of the 1999 congress on evolutionary computation, Washington DC, July 1999, pp. 1945-1949.

[16] M. Clerc and J. Kennedy, "The particle swarm-explosion, stability, and convergence in a multidimensional complex space," IEEE Transactions on Evolutionary Computation, vol. 6, pp. 58-73, 2002.

[17] I. C. Trelea, "The particle swarm optimization algorithm: Convergence analysis and parameter selection,” Information Processing Letters, vol. 85, pp. 317-325, 2003.

[18] R. M. May, "Simple mathematical models with very complicated dynamics,” Nature, vol.261, pp. 459-467, 1976.

[19] D. E. Knuth, "The Art of Computer Programming, Seminumerical Algorithms,” Third ed., vol. 2, Addison-Wesley, 1997, pp. 10-26.

[20] D. Kuo, "Chaos and its computing paradigm," IEEE Potentials Magazine, vol. 24, pp. 13-15, 2005. 